\title{
Fluid Reaction Force on Solids
}

\section{Kern E. Kenyon}

4632 North Lane, Del Mar, CA, USA

Correspondence to: Kern E. Kenyon, kernken@aol.com

Keywords: Reaction Forces, Fluid/Solid Interactions

Received: September 28, $2018 \quad$ Accepted: October 17, $2018 \quad$ Published: October 20, 2018

Copyright $\odot 2018$ by author and Scientific Research Publishing Inc.

This work is licensed under the Creative Commons Attribution International License (CC BY 4.0).

http://creativecommons.org/licenses/by/4.0/

\section{(c) $\underset{\mathrm{BY}}{\mathrm{BY}}$ Open Access}

\section{ABSTRACT}

Steady flow past a circular arc wing produces a vertical lift force, which has been calculated before, but it also causes two horizontal reaction forces, equal and opposite, due to Newton's third law: the accelerated and decelerated flows act back on the top surfaces of the wing. Reaction forces are computed here and compared with the lift force. The magnitude of the two perpendicular forces are equal when the ratio of the maximum thickness of the wing is about 1/5 the length of the wing's flat bottom surface. An example of an asymmetric wing is discussed and the net reaction force is calculated, which is always directed horizontally away from the top surface of the wing that has the greater mean slope. Based on these results, it is predicted that a surface ship should go more easily through the water if the bow were blunt and the stern pointed, just the opposite configuration of what is commonly found traversing lakes and oceans. Surface gravity waves are argued not to change this conclusion in general.

\section{INTRODUCTION}

When a fluid accelerates along the smooth surface of a solid body, due to the presence of the body in a steady flow, there is an equal but opposite "reaction" force of the fluid back on the body, in accordance with Newton's third law of motion. To my knowledge, no fluid dynamics text contains this concept. A description of the reaction force has been published [1] with the help of certain assumptions. One of the central assumptions is related to the still unknown detailed behavior of the fluid in the vicinity of the solid. For example, if a steady flow of fluid along a flat solid surface of a wall encounters a single bump, protruding into the flow, exactly how does the perturbed motion at the top of the bump decay with increasing distance away from the bump?

In the earlier study, the assumption was made that the perturbed velocity decays exponentially with distance from the bump such that the e-folding scale is only proportional to the bump's length for a thin bump: height over length of the bump is much less than one. This was done by analogy with the exponential depth decay rate of all properties of the surface gravity wave, with the e-folding scale being propor- 
tional only to the wavelength, as a consequence of the application of the mathematical irrotational theory, even though no solid surfaces were involved in that example.

If the potential flow method is applied to a cylinder immersed in a steadily moving fluid, it is found that the velocity above and below the cylinder falls off as the inverse square of the distance from the top and bottom of the cylinder. [Curiously for the sphere the decay rate is the inverse cube of the distance.] Not in either case has an observation been held up against the decay rate prediction, not for the cylinder and not for the surface gravity wave.

There is available now a different, more physical, theoretical approach to understanding the decay rates of perturbed flows, with or without solid boundaries, and it is very likely that the new and the old theories will not turn out to be in complete agreement. Start by balancing the centrifugal force on a fluid particle negotiating a curved streamline over a bump in the wall, with a cross-stream pressure gradient. Together with Bernoulli's equation along a streamline there are two equations in two unknowns: pressure and velocity. Both equations are nonlinear, but by elimination of one variable, one equation in one unknown is made, which is a linear first order ordinary differential equation, for both pressure and velocity (although there is a slight but significant difference between the two governing equations involving a factor of 2). To obtain a complete solution of the governing equation the non-constant coefficient needs to be supplied from either measurements or from another theory. The non-constant coefficient is the radius of curvature of the streamlines as a function of distance normal to the streamlines. Because of an integration in the solution over this distance, the end result for pressure or velocity is not sensitive to the particular choice made for the radius of curvature function.

Inching toward practicality the force balance method has been used to approximate the lift force $L$ on a circular arc wing in a uniform flow [2], resulting in

$$
L=\frac{1.3 \rho U^{2}}{R_{0}}
$$

where $L$ is the lift force at the top of the circular arc wing, $\rho$ is the constant fluid density, $U$ is the flow speed far from the wing, and $R_{0}$ is the radius of the circular arc. For comparison the reaction force on both front and back top faces of the circular arc wing are computed next, and it will be seen under what conditions the two different (perpendicular) forces can be equal in magnitude.

\section{REACTION FORCES: CIRCULAR ARC WING}

On the front top face of the circular arc wing the increase in horizontal linear momentum of the fluid $\Delta M$ is

$$
\Delta M=\rho(V-U)
$$

where $V$ is the flow speed at the top of the wing and $U$ is the uniform flow speed far away from the wing. From a previous calculation in reference [2] comes

$$
V-U=\frac{3.4 U h}{R_{0}}
$$

where $h$ is the maximum vertical thickness of the wing.

Then the positive time rate of change in the momentum $N=\Delta M / \Delta T$ is approximately

$$
N=\frac{\Delta M}{\Delta T}=\frac{\Delta M}{l / 2 U}=\frac{6.8 \rho U^{2} h}{R_{0} l}
$$

where $1 / 2$ is half the cord or half the length $l$ of the bottom flat face of the wing. The reaction force is then $-N$ and points horizontally toward the oncoming flow. Now the comparison with the lift force becomes

$$
N=5.2 \frac{h}{l} L
$$


showing that the reaction force magnitude is the same order as that of the lift force if the maximum thickness of the wing is not too small compared to its cord length.

\section{REACTION FORCES: ASYMMETRIC WING}

In the above example with front to back symmetry, the front pointing and back pointing reaction forces have equal magnitudes. When front to back asymmetry occurs on the top surface of the wing, that will not be the case and a net reaction force will occur. Consider that the front top face of the wing is the same circular arc, a piece a bit more than $1 / 2$, and that the back face is a straight line connecting the circular arc piece and sloping down to the horizontal flat bottom of the wing with total length $l$. But now $l=l_{1}+l_{2}$, where $l_{1}$ is the length from the front tip of the wing to just under the top of the wing, $l_{2}$ is the length from there to the back tip, and $l_{2} \geq l_{1}$.

Immediately the net reaction force can be written down by following the above procedure separately for the front and back faces of the wing and then subtracting the result for the back face from that on the front face.

$$
N_{\text {net }}=\frac{6.8 \rho U^{2} h}{R_{0}}\left(\frac{1}{l_{1}}-\frac{1}{l_{2}}\right)
$$

And it points horizontally in the direction of the oncoming flow.

\section{DISCUSSION}

Nature's blunt front method of making it easier for solids to move through fluids has many examples, a few of which are listed in the earlier paper [1]. However, man still chooses not to follow along when he designs most surface ships that travel the seas and shoots bullets through the air because in both cases they have pointed fronts.

From a well done photo book of many different kinds of ducks, where several pictures were taken from above straight down, I traced the waterlines of a few species. They all had a fat tear drop shape with the point in the rear. This example is mentioned because in the course of crossing ponds and lakes ducks must deal with waves as well as currents. Apparently it is the ocean waves that cause naval architects to believe that the bows should be sharply pointed.

Consider the gray whale, which migrates thousands of miles between the Gulf of Alaska and Baja California and back again every year. Since it breathes air, the whale never leaves the zone of influence of the surface gravity waves. Millions of years of evolution have helped develop its blunt front/pointed rear shape.

To attempt a physical explanation for why a surface ship with a blunt front would not be inconvenienced by encountering waves in most cases, remember the fact that the net reaction force on an asymmetric solid in a uniform flow is the same, in magnitude and direction, for either way the flow passes by it: the net force always points away from the blunt front [1]. So imagination will provide a good reason why a ship with a pointed bow and blunt stern is worse off than if it were turned 180 degrees around, even without consideration of the waves.

Suppose a ship is in an oscillating flow. Then the same truth holds: the net reaction force will oscillate in magnitude but never change direction. From that comes the deduction that a blunt front ship headed into the waves should have no difficulty in two limiting situations at least: the wavelength is much greater than or much less than the length of the ship. For wavelengths comparable to the ship's length a more thorough investigation might be needed.

\section{CONCLUSION}

Reaction forces of fluids passing by solid bodies are brought into focus by means of calculations in a specific model and a comparison with the better known lift force. A circular arc wing in a uniform flow 
experiences a vertical lift force and two horizontal, equal and opposite, reaction forces that arise from Newton's third law. When a fluid accelerates (or decelerates) along the surface of a solid body, the fluid acts back on the surface. Magnitudes of the two perpendicular forces are equal if the maximum thickness of the wing is about $1 / 5$ of the length of the wing's flat bottom surface, according to the computation. An asymmetric wing example has a net reaction force that points horizontally away from that part of the wing whose top surface has the larger mean slope, the magnitude of which is derives algebraically. These results lead to the prediction that a surface ship going through water would use less fuel if the bow were blunt and the stern pointed rather than the other way around. An argument is presented to show that the presence of surface gravity waves should not change this conclusion in most cases.

\section{ACKNOWLEDGEMENTS}

Part of this manuscript was stimulated by discussions with Wilton Sturges.

\section{CONFLICTS OF INTEREST}

The author declares no conflicts of interest regarding the publication of this paper.

\section{REFERENCES}

1. Kenyon, K.E. (2006) Reaction Forces of Fluid Flows on Solid Boundaries. Physics Essays, 19, 507-516. https://doi.org/10.4006/1.3028856

2. Kenyon, K.E. (2017) Lift Force on a Circular Arc Wing. Natural Science, 9, 351-354.

https://doi.org/10.4236/ns.2017.910033 